Korean J. Math. 19 (2011), No. 4, pp. 381-390

\title{
A STUDY ON THE CONTRACTED ES CURVATURE TENSOR IN $g-E S X_{n}$
}

\author{
IN Ho HWANG
}

\begin{abstract}
This paper is a direct continuation of [1]. In this paper we derive tensorial representations of contracted ES curvature tensors of $g-E S X_{n}$ and prove several generalized identities involving them. In particular, a variation of the generalized Bianchi's identity in $g-E S X_{n}$, which has a great deal of useful physical applications, is proved in Theorem (2.9).
\end{abstract}

\section{Preliminaries}

This paper is a direct continuation of our previous paper [1], which will be denoted by I in the present paper. All considerations in this paper are based on our results and symbolism of $\mathrm{I}([1],[2],[3],[4],[5],[6],[7],[8],[9])$. Whenever necessary, these results will be quoted in the text. In this section, we introduce a brief collection of basic concepts, notations, and results of I, which are frequently used in the present paper.

(a) Let $X_{n}$ be a generalized $n$-dimensional Riemannian manifold referred to a real coordinate system $x^{\nu}$, which obeys the coordinate transformations $x^{\nu} \rightarrow x^{\nu^{\prime}}$ for which

$$
\operatorname{det}\left(\frac{\partial x^{\prime}}{\partial x}\right) \neq 0
$$

In $n-g-U F T$ the manifold $X_{n}$ is endowed with a real nonsymmetric tensor $g_{\lambda \mu}$, which may be decomposed into its symmetric

\footnotetext{
Received October 7, 2011. Revised December 8, 2011. Accepted December 10, 2011.

2000 Mathematics Subject Classification: 83E50, 83C05, 58A05.

Key words and phrases: ES-manifold, ES-curvature tnesor, contracted EScurvature tensor.

This research was supported by University of Incheon Research Grant, 2010-2011.
} 
part $h_{\lambda \mu}$ and skew-symmetric part $k_{\lambda \mu}$ :

$$
g_{\lambda \mu}=h_{\lambda \mu}+k_{\lambda \mu} \text {. }
$$

where

$$
\mathfrak{g}=\operatorname{det}\left(g_{\lambda \mu}\right) \neq 0, \quad \mathfrak{h}=\operatorname{det}\left(h_{\lambda \mu}\right) \neq 0, \quad \mathfrak{k}=\operatorname{det}\left(k_{\lambda \mu}\right) .
$$

In virtue of (1.3) we may define a unique tensor $h^{\lambda \nu}$ by

$$
h_{\lambda \mu} h^{\lambda \nu}=\delta_{\mu}^{\nu}
$$

which together with $h_{\lambda \mu}$ will serve for raising and/or lowering indices of tensors in $X_{n}$ in the usual manner. There exists a unique tensor $* g^{\lambda \nu}$ satisfying

$$
g_{\lambda \mu}{ }^{*} g^{\lambda \nu}=g_{\mu \lambda}{ }^{*} g^{\nu \lambda}=\delta_{\mu}^{\nu} .
$$

It may be also decomposed into its symmetric part ${ }^{*} h^{\lambda \nu}$ and skewsymmetric part ${ }^{*} k^{\lambda \nu}$ :

$$
{ }^{*} g^{\lambda \nu}={ }^{*} h^{\lambda \nu}+{ }^{*} k^{\lambda \nu} .
$$

The manifold $X_{n}$ is connected by a general real connection $\Gamma_{\lambda}{ }^{\nu} \mu$ with the following transformation rule:

$$
\Gamma_{\lambda^{\prime}}^{\nu^{\prime}}{ }_{\mu^{\prime}}=\frac{\partial x^{\nu^{\prime}}}{\partial x^{\alpha}}\left(\frac{\partial x^{\beta}}{\partial x^{\lambda^{\prime}}} \frac{\partial x^{\gamma}}{\partial x^{\mu^{\prime}}} \Gamma_{\beta}{ }^{\alpha}{ }_{\gamma}+\frac{\partial^{2} x^{\alpha}}{\partial x^{\lambda^{\prime}} \partial x^{\mu^{\prime}}}\right) .
$$

It may also be decomposed into its symmetric part $\Lambda_{\lambda}{ }_{\mu}{ }_{\mu}$ and its skew-symmetric part $S_{\lambda \nu}{ }^{\nu}$, called the torsion tensor of $\Gamma_{\lambda}{ }^{\nu}{ }_{\mu}$ :

$\left.\Gamma_{\lambda}{ }^{\nu}{ }_{\mu}=\Lambda_{\lambda}{ }^{\nu} \mu+S_{\lambda \mu}{ }^{\nu} ; \quad \Lambda_{\lambda}{ }^{\nu}{ }_{\mu}=\Gamma_{\left(\lambda^{\nu} \mu\right)} ; \quad S_{\lambda \mu}{ }^{\nu}=\Gamma_{[\lambda}{ }^{\nu} \mu\right]$.

A connection $\Gamma_{\lambda}{ }^{\nu}{ }_{\mu}$ is said to be Einstein if it satisfies the following system of Einstein's equations:

$$
\partial_{\omega} g_{\lambda \mu}-\Gamma_{\lambda}{ }^{\alpha}{ }_{\omega} g_{\alpha \mu}-\Gamma_{\omega}{ }^{\alpha}{ }_{\mu} g_{\lambda \alpha}=0 .
$$

or equivalently

$$
D_{\omega} g_{\lambda \mu}=2 S_{\omega \mu}{ }^{\alpha} g_{\lambda \alpha} .
$$

where $D_{\omega}$ is the symbolic vector of the covariant derivative with respect to $\Gamma_{\lambda^{\nu}}{ }_{\mu}$. In order to obtain $g_{\lambda \mu}$ involved in the solution for $\Gamma_{\lambda}{ }^{\nu}{ }_{\mu}$ in (1.9), certain conditions are imposed. These conditions may be condensed to

$$
S_{\lambda}=S_{\lambda \alpha}{ }^{\alpha}=0, \quad R_{[\mu \lambda]}=\partial_{[\mu} Y_{\lambda]}, \quad R_{(\mu \lambda)}=0 .
$$


A study on the contracted ES curvature tensor in $g-E S X_{n}$

where $Y_{\lambda}$ is an arbitrary vector, and

$$
\left.R_{\omega \mu \lambda}{ }^{\nu}=2\left(\partial_{[\mu} \Gamma_{\left.|\lambda|^{\nu} \omega\right]}+\Gamma_{\alpha}{ }^{\nu}{ }_{[\mu} \Gamma_{|\lambda|}{ }^{\alpha} \omega\right]\right) .
$$

If the system (1.10) admits a solution $\Gamma_{\lambda}{ }^{\nu}{ }_{\mu}$, it must be of the form (Hlavatý, 1957)

$$
\Gamma_{\lambda}{ }^{\nu}=\left\{\begin{array}{l}
\nu \\
\lambda \mu
\end{array}\right\}+S_{\lambda \mu}{ }^{\nu}+U^{\nu}{ }_{\lambda \mu} .
$$

where $U_{\lambda \mu}^{\nu}=2 h^{\nu \alpha} S_{\alpha\left(\lambda^{\beta}\right.} k_{\mu) \beta}$ and $\left\{\begin{array}{l}\nu \\ \lambda \mu\end{array}\right\}$ are Christoffel symbols defined by $h_{\lambda \mu}$.

(b) Some notations and results The following quantities are frequently used in our further considerations:

$$
{ }^{(0)} k_{\lambda}{ }^{\nu}=\delta_{\lambda}^{\nu},{ }^{(p)} k_{\lambda}{ }^{\nu}=k_{\lambda}{ }^{\alpha}{ }^{(p-1)} k_{\alpha}{ }^{\nu} \quad(p=1,2, \cdots) .
$$

In $X_{n}$ it was proved in [5] that

(1.17) $K_{0}=1, K_{n}=k$ if $n$ is even, and $\mathrm{K}_{\mathrm{p}}=0$ if $\mathrm{p}$ is odd.

$$
\begin{aligned}
\mathfrak{g} & =\mathfrak{h}\left(1+K_{1}+K_{2}+\cdots+K_{n}\right) \\
\text { or } g & =1+K_{1}+K_{2}+\cdots+K_{n} .
\end{aligned}
$$

$$
\sum_{s=0}^{n-\sigma} K_{s}{ }^{(n-s+p)} k_{\lambda}{ }^{\nu}=0 \quad(p=0,1,2, \cdots) .
$$

We also use the following useful abbreviations for an arbitrary vector $Y$, for $p=1,2,3, \cdots$ :

$$
\begin{aligned}
& { }^{(p)} Y_{\lambda}={ }^{(p-1)} k_{\lambda}{ }^{\alpha} Y_{\alpha}, \\
& { }^{(p)} Y^{\nu}={ }^{(p-1)} k_{\alpha}^{\nu} Y^{\alpha} .
\end{aligned}
$$

(c) n-dimensional ES manifold $E S X_{n}$ 
In this subsection, we display an useful representation of the $E S$ connection in $n-g$-UFT.

Definition 1.1. A connection $\Gamma_{\lambda}{ }^{\nu}{ }_{\mu}$ is said to be semi-symmetric if its torsion tensor $S_{\lambda \mu}{ }^{\nu}$ is of the form

$$
S_{\lambda \mu}{ }^{\nu}=2 \delta_{[\lambda}^{\nu} X_{\mu]}
$$

for an arbitrary non-null vector $X_{\mu}$.

A connection which is both semi-symmetric and Einstein is called an $E S$ connection. An $n$-dimensional generalized Riemannian manifold $X_{n}$, on which the differential geometric structure is imposed by $g_{\lambda \mu}$ by means of an $E S$ connection, is called an $n$-dimensional $E S$ manifold. We denote this manifold by $g-E S X_{n}$ in our further considerations.

TheOrem 1.2. Under the condition (1.22), the system of equations (1.10) is equivalent to

$$
\Gamma_{\lambda}{ }^{\nu}{ }_{\mu}=\left\{\begin{array}{c}
\nu \\
\lambda \mu
\end{array}\right\}+2 k_{(\lambda}^{\nu} X_{\mu)}+2 \delta_{[\lambda}^{\nu} X_{\mu]} .
$$

Proof. Substituting (1.22) for $S_{\lambda \mu}{ }^{\nu}$ into (1.13), we have the representation (1.23).

Theorem 1.3. In $g-E S X_{n}$, the following relations hold for $p, q=$ $1,2,3, \cdots$ :

$$
\begin{gathered}
S_{\lambda}=(1-n) X_{\lambda}, \\
U_{\lambda}=\frac{1}{2} \partial_{\lambda} l n \mathfrak{g}, \\
{ }^{(p+1)} S_{\lambda}=(1-n)^{(p)} U_{\lambda}, \\
{ }^{(p)} U_{\alpha}{ }^{(q)} X^{\alpha}=0 \quad \text { if } \quad p+q-1 \quad \text { is } \quad \text { odd }, \\
D_{\lambda} X_{\mu}=\nabla_{\lambda} X_{\mu}, \\
D_{[\lambda} X_{\mu]}=\nabla_{[\lambda} X_{\mu]}=\partial_{[\lambda} X_{\mu]}, \\
\nabla_{[\lambda} U_{\mu]}=0, \quad D_{[\lambda} U_{\mu]}=2 U_{[\lambda} X_{\mu]}=2^{(2)} X_{[\lambda} X_{\mu]},
\end{gathered}
$$

where $\nabla_{\omega}$ is the symbolic vector of the covariant derivative with respect to the Christoffel symbols defined by $h_{\lambda \mu}$. 
TheOREM 1.4. In $g-E S X_{n}$ under the present conditions, the ES curvature tensor $R_{\omega \mu \lambda}{ }^{\nu}$ may be given by

$$
R_{\omega \mu \lambda}^{\nu}=L_{\omega \mu \lambda}^{\nu}+M_{\omega \mu \lambda}^{\nu}+N_{\omega \mu \lambda}^{\nu},
$$

where

$$
\begin{gathered}
L_{\omega \mu \lambda}{ }^{\nu}=2\left(\partial_{[\mu}\left\{\begin{array}{c}
\nu \\
\omega] \lambda
\end{array}\right\}+\left\{\begin{array}{c}
\nu \\
\alpha[\mu
\end{array}\right\}\left\{\begin{array}{c}
\alpha \\
\omega] \lambda
\end{array}\right\}\right), \\
M_{\omega \mu \lambda}{ }^{\nu}=2\left(\delta_{\lambda}^{\nu} \partial_{[\mu} X_{\omega]}+\delta_{[\mu}^{\nu} \nabla_{\omega]} X_{\lambda}+\nabla_{[\mu} U^{\nu}{ }_{\omega] \lambda}\right), \\
N_{\omega \mu \lambda}{ }^{\nu}=2\left(\delta_{[\omega}^{\nu} X_{\mu]} X_{\lambda}+{ }^{(2)} X_{\lambda} k_{[\mu}{ }^{\nu} X_{\omega]}\right) .
\end{gathered}
$$

THEOREM 1.5. (Generalized Bianchi's identity in $g-E S X_{n}$ ) Under the present conditions, the ES curvature tensor $R_{\omega \mu \lambda}{ }^{\nu}$ of $g-E S X_{n}$ satisfies the following identity:

$$
D_{[\epsilon} R_{\omega \mu] \lambda}^{\nu}=-4 X_{[\epsilon} L_{\omega \mu] \lambda}^{\nu}+O_{[\epsilon \omega \mu] \lambda}^{\nu},
$$

where

$$
\begin{aligned}
\frac{1}{8} O_{\epsilon \omega \mu \lambda}{ }^{\nu} & =\delta_{\lambda}^{\nu} X_{\epsilon} \partial_{\omega} X_{\mu}+X_{\epsilon} \delta_{\omega}^{\nu} \nabla_{\mu} X_{\lambda} \\
& +X_{\epsilon} \nabla_{\omega} U^{\nu}{ }_{\mu \lambda}+X_{\epsilon} \delta_{\mu}^{\nu} X_{\omega} X_{\lambda}+{ }^{(2)} X_{\lambda} X_{\epsilon} k_{\omega}{ }^{\nu} X_{\mu}
\end{aligned}
$$

\section{The contracted ES curvature tensors in $g-E S X_{n}$}

This section is devoted to the study of the contracted $n$-dimensional ES curvature tensors, defined by the ES connection in $g$-UFT under the present conditions, and of some useful identities involving them.

The tensors

$$
R_{\mu \lambda}=R_{\alpha \mu \lambda}^{\alpha}, \quad V_{\omega \mu}=R_{\omega \mu \alpha}^{\alpha}
$$

are called the first and second contracted ES curvature tensors of the ES connection $\Gamma_{\lambda}{ }^{\nu}{ }_{\mu}$, respectively. We see in the following two theorems that they appear as functions of the vectors $X_{\lambda}, S_{\lambda}, U_{\lambda}$, and hence also as functions of $g_{\lambda \mu}$ and its first two derivatives in virtue of $(1.24,25)$ and (1.31). 
TheOREM 2.1. The first contracted ES curvature tensor $R_{\mu \lambda}$ in $g-$ $E S X_{n}$ may be given by

$$
\begin{aligned}
R_{\mu \lambda}=L_{\mu \lambda}+2 \partial_{[\mu} X_{\lambda]} & +\nabla_{\mu} T_{\lambda}-\nabla_{\alpha} U^{\alpha}{ }_{\mu \lambda} \\
& +(n-1) X_{\mu} X_{\lambda}+U_{\mu} U_{\lambda},
\end{aligned}
$$

where

$$
L_{\mu \lambda}=L_{\alpha \mu \lambda}{ }^{\alpha},
$$

$$
T_{\lambda \mu}{ }^{\nu}=S_{\lambda \mu}{ }^{\nu}+U_{\lambda \mu}^{\nu}, \quad T_{\lambda}=T_{\lambda \alpha}{ }^{\alpha}=S_{\lambda}+U_{\lambda} .
$$

Proof. Putting $\omega=\nu=\alpha$ in (1.31) and making use of (2.3), we have

$$
R_{\mu \lambda}=L_{\mu \lambda}+M_{\alpha \mu \lambda}^{\alpha}+N_{\alpha \mu \lambda}^{\alpha} .
$$

In virtue of $(1.24,25)$, it follows from $(1.33)$ that

$$
\begin{aligned}
M_{\alpha \mu \lambda}{ }^{\alpha} & =2 \partial_{[\mu} X_{\lambda]}+(1-n) \nabla_{\mu} X_{\lambda}+\nabla_{\mu} U_{\lambda}-\nabla_{\alpha} U^{\alpha}{ }_{\mu \lambda} \\
& =2 \partial_{[\mu} X_{\lambda]}+\nabla_{\mu} T_{\lambda}-\nabla_{\alpha} U^{\alpha}{ }_{\mu \lambda} .
\end{aligned}
$$

On the other hand, in virtue of (1.25) the relation (1.34) gives

$$
\begin{aligned}
N_{\alpha \mu \lambda}{ }^{\alpha} & =(n-1) X_{\mu} X_{\lambda}+{ }^{(2)} X_{\mu}^{(2)} X_{\lambda}-{ }^{(2)} X_{\lambda} X_{\mu} k_{\alpha}{ }^{\alpha} \\
& =(n-1) X_{\mu} X_{\lambda}+U_{\mu} U_{\lambda} .
\end{aligned}
$$

Our assertion follows immediately from (2.5), (2.6) and (2.7).

THEOREM 2.2. The second contracted ES curvature tensor $V_{\omega \mu}$ in $g-E S X_{n}$ is a curl of the vector $S_{\lambda}$. That is,

$$
V_{\omega \mu}=2 \partial_{[\omega} S_{\mu]} .
$$

Proof. Putting $\lambda=\nu=\alpha$ in (1.31), we have

$$
V_{\omega \mu}=L_{\omega \mu \alpha}{ }^{\alpha}+M_{\omega \mu \alpha}{ }^{\alpha}+N_{\omega \mu \alpha}{ }^{\alpha} .
$$

In virtue of $(1.11)$ and $(1.24,25,30)$, the relations $(1.32,33,34)$ give

$$
\begin{gathered}
L_{\omega \mu \alpha}{ }^{\alpha}=N_{\omega \mu \alpha}{ }^{\alpha}=0 \\
M_{\omega \mu \alpha}{ }^{\alpha}=2(1-n) \partial_{[\omega} X_{\mu]}+2 \nabla_{[\mu} U_{\omega]}=2(1-n) \partial_{[\omega} X_{\mu]}=2 \partial_{[\omega} S_{\mu]}
\end{gathered}
$$

which together with (2.9) proves our assertion. 
Theorem 2.3. The tensor $R_{\mu \lambda}$ is symmetric when $n=3$.

Proof. The relation (2.2) may be written as

$$
\begin{gathered}
R_{\mu \lambda}=L_{\mu \lambda}+(3-n) \nabla_{\mu} X_{\lambda}-2 \nabla_{(\mu} X_{\lambda)}+\nabla_{\mu} U_{\lambda} \\
-\nabla_{\alpha} U^{\alpha}{ }_{\mu \lambda}+(n-1) X_{\mu} X_{\lambda}+U_{\mu} U_{\lambda},
\end{gathered}
$$

where use has been made of $(1.24,29)$ and $(2.4)$. Hence, in virtue of $(1.29,30)$ we have $R_{[\mu \lambda]}=0$ if and only if $(3-n) \nabla_{[\mu} X_{\lambda]}=(3-n) \partial_{[\mu} X_{\lambda]}=$ 0 .

REMARK 2.4. In the proof of the Theorem (2.3), we excluded the case that $\partial_{[\mu} X_{\lambda]}=0$, because we assumed that $X_{\lambda}$ is not a gradient vector in the definition of semi-symmetric connection in (1.22). In fact, the assumption that $X_{\lambda}$ is not a gradient vector is essential in the discussions of the field equations in $g-E S X_{n}$.

THEOREM 2.5. The contracted ES curvature tensors in $g-E S X_{n}$ are related by

$$
2 R_{[\mu \lambda]}=4 \partial_{[\mu} X_{\lambda]}+V_{\mu \lambda} .
$$

Proof. In virtue of $(1.24,29,30)$, the relation (2.11) may be proved from (2.10) as in the following way:

$$
\begin{aligned}
2 R_{[\mu \lambda]} & =2(3-n) \partial_{[\mu} X_{\lambda]} \\
& =2(1-n) \partial_{[\mu} X_{\lambda]}+4 \partial_{[\mu} X_{\lambda]} \\
& =2 \partial_{[\mu} S_{\lambda]}+4 \partial_{[\mu} X_{\lambda]} \\
& =V_{\mu \lambda}+4 \partial_{[\mu} X_{\lambda]} .
\end{aligned}
$$

Our next task is to obtain a generalization of the classical identity

$$
\nabla_{\alpha} E_{\mu}^{\alpha}=0
$$

where

$$
L=h^{\alpha \beta} L_{\alpha \beta}, \quad E_{\mu}{ }^{\nu}=L_{\mu}{ }^{\nu}-\frac{1}{2} \delta_{\mu}^{\nu} L .
$$


REMARK 2.6. The tensor $E_{\mu}{ }^{\nu}$ is called the Einstein tensor. This tensor has a great deal of applications in physics. It is of fundamental importance since its divergence vanishes identically as we see in (2.13).

In our further considerations, the quantities

$$
R=h^{\alpha \beta} R_{\alpha \beta}, \quad G_{\mu}{ }^{\nu}=R_{\mu}{ }^{\nu}-\frac{1}{2} \delta_{\mu}^{\nu} R
$$

will be referred to ES curvature invariant and ES Einstein tensor of $g-E S X_{n}$, respectively. The tensor $G_{\mu}{ }^{\nu}$ is the generalized concept of $E_{\mu}{ }^{\nu}$. First of all, we need the following two theorems in order to generalize the identity (2.13) in $g-E S X_{n}$.

TheOREM 2.7. In $g-E S X_{n}$, we have

$$
D_{\omega} h^{\lambda \mu}=2 X^{(\lambda} g_{\omega}^{\mu)}-2 X_{\omega} h^{\lambda \mu} .
$$

Proof. Substituting (1.22) into (1.10) for $S_{\omega \alpha}{ }^{\nu}$ and making use of (1.2) and (1.21), the relations (2.16) follows as in the following way:

$$
\begin{aligned}
D_{\omega} h^{\lambda \mu} & =2 S_{\omega(\alpha}^{\gamma} g_{\beta) \gamma} h^{\lambda \alpha} h^{\mu \beta} \\
& =2\left(\delta_{[\omega}^{\gamma} X_{\alpha]} g_{\beta \gamma}+\delta_{[\omega}^{\gamma} X_{\beta]} g_{\alpha \gamma}\right) h^{\lambda \alpha} h^{\mu \beta} \\
& =2\left(g_{\beta[\omega} X_{\alpha]}+g_{\alpha[\omega} X_{\beta]}\right) h^{\lambda \alpha} h^{\mu \beta} \\
& =2 X^{(\lambda} g_{\omega}^{\mu)}-2 X_{\omega} h^{\lambda \mu} .
\end{aligned}
$$

THEOREM 2.8. In $g-E S X_{n}$, we have

$$
\begin{aligned}
& R=L+(1-n) \nabla_{\alpha} X^{\alpha}+\nabla_{\alpha} U^{\alpha}+(n-1) X \\
&+U-\nabla_{\gamma} U_{\alpha \beta}^{\gamma}, \\
& D_{\alpha} R_{\mu}{ }^{\alpha}=\nabla_{\alpha} R_{\mu}{ }^{\alpha}+\left(U_{\alpha}-n X_{\alpha}\right) R_{\mu}{ }^{\alpha}+R X_{\mu}-U^{\alpha} R_{\alpha \mu},
\end{aligned}
$$

where

$$
X=X_{\alpha} X^{\alpha}, \quad U=U_{\alpha} U^{\alpha} .
$$

Proof. In virtue of $(2.14),(2.15)$ and $(1.24,29)$, the representation (2.17) follows from (2.2). On the other hand, the representation (2.18) 
may be proved as in the following way in virtue of $(1.13),(1.22),(1.24),(1.25)$ and (2.15):

$$
\begin{aligned}
D_{\alpha} R_{\mu}{ }^{\alpha} & =\partial_{\alpha} R_{\mu}{ }^{\alpha}+\Gamma_{\beta \alpha}^{\alpha} R_{\mu}{ }^{\beta}-\Gamma_{\mu \alpha}^{\beta} R_{\beta}{ }^{\alpha} \\
& =\nabla_{\alpha} R_{\mu}{ }^{\alpha}+\left(S_{\beta}+U_{\beta}\right) R_{\mu}{ }^{\beta}-S_{\mu \alpha}{ }^{\beta} R_{\beta}{ }^{\alpha}-U^{\beta}{ }_{\mu \alpha} R_{\beta}{ }^{\alpha} \\
& =\nabla_{\alpha} R_{\mu}{ }^{\alpha}+(1-n) X_{\alpha}+U_{\alpha} R_{\mu}{ }^{\alpha}+2 \delta_{[\alpha}^{\beta} X_{\mu]} R_{\beta}{ }^{\alpha}-U^{\beta}{ }_{\mu \alpha} R_{\beta}{ }^{\alpha} \\
& =\nabla_{\alpha} R_{\mu}{ }^{\alpha}+\left(U_{\alpha}-n X_{\alpha}\right) R_{\mu}{ }^{\alpha}+R X_{\mu}-U^{\beta}{ }_{\mu \alpha} R_{\beta}{ }^{\alpha} \\
& =\nabla_{\alpha} R_{\mu}{ }^{\alpha}+\left(U_{\alpha}-n X_{\alpha}\right) R_{\mu}{ }^{\alpha}+R X_{\mu}-U^{\alpha} R_{\alpha \mu} .
\end{aligned}
$$

Now we are ready to prove the following generalization of (2.13).

THEOREM 2.9. (A variation of the generalized Bianchi's identity in $\left.g-E S X_{n}\right)$. The ES Einstein tensor $G_{\mu}{ }^{\nu}$ satisfies the following identity in $g-E S X_{n}$ :

$$
D_{\alpha} G_{\mu}{ }^{\alpha}=P_{\mu}-\frac{1}{2} \partial_{\mu} Q
$$

where

$(2.21) P_{\mu}=\nabla_{\alpha}\left(R_{\mu}{ }^{\alpha}-L_{\mu}{ }^{\alpha}\right)+\left(U_{\alpha}-n X_{\alpha}\right) R_{\mu}{ }^{\alpha}+R X_{\mu}-U^{\alpha} R_{\alpha \mu}$,

$(2.22) Q=(1-n) \nabla_{\alpha} X^{\alpha}+\nabla_{\alpha} U^{\alpha}+U+(n-1) X-U_{\gamma} U^{\gamma}{ }_{\alpha \beta}$.

Proof. The relation (2.15) gives

$$
\begin{aligned}
& D_{\alpha} G_{\mu}{ }^{\alpha}=D_{\alpha}\left(R_{\mu}{ }^{\alpha}-\frac{1}{2} \delta_{\mu}^{\alpha} R\right) \\
& =\nabla_{\alpha}\left(R_{\mu}{ }^{\alpha}-L_{\mu}{ }^{\alpha}\right)+\left(U_{\alpha}-n X_{\alpha}\right) R_{\mu}{ }^{\alpha}+R X_{\mu}-U^{\alpha} R_{\alpha \mu} \\
& -\frac{1}{2} \partial_{\mu}\left[(1-n) \nabla_{\alpha} X^{\alpha}+\nabla_{\alpha} U^{\alpha}+U+(n-1) X-U_{\gamma} U^{\gamma}{ }_{\alpha \beta}\right] .
\end{aligned}
$$

The proof of the identity (2.20) immediately follows by substituting $(2.17,18)$ into $(2.23)$ and making use of $(2.21,22)$.

\section{References}

[1] Hwang, I.H., On the ES curvature tensor in $g-E S X_{n}$, Korean J. Math. 19 (1) (2011), 25-32.

[2] Hwang, I.H., A study on the recurrence relations and vectors $X_{\lambda}, S_{\lambda}$ and $U_{\lambda}$ in $g-E S X_{n}$, Korean J. Math. 18 (2) (2010), 133-139. 
[3] Hwang, I.H., A study on the geometry of 2-dimensional RE-manifold $X_{2}$, J. Korean Math. Soc., 32 (2) (1995), 301-309.

[4] Hwang, I.H., Three- and Five- dimensional considerations of the geometry of Einstein's g-unified field theory, Int. J. Theor. Phys. 27 (9) (1988), 1105-1136.

[5] Chung, K.T., Einstein's connection in terms of ${ }^{*} g^{\lambda \nu}$, Nuovo cimento Soc. Ital. Fis. B, 27 (X) (1963), 1297-1324.

[6] Datta, D.k., Some theorems on symmetric recurrent tensors of the second order, Tensor (N.S.) 15 (1964), 1105-1136.

[7] Einstein, A., The meaning of relativity, Princeton University Press,1950.

[8] Hlavatý, V., Geometry of Einstein's unified field theory, Noordhoop Ltd., 1957.

[9] Mishra, R.S., n-dimensional considerations of unified field theory of relativity, Tensor(N.S.) 9 (1959), 217-225.

Department of Mathematics

University of Incheon

Incheon 406-772, Korea

E-mail: ho818@incheon.ac.kr 